Check for updates

Cite this: RSC Adv., 2018, 8, 17254

\title{
Photoluminescence mechanism and applications of Zn-doped carbon dots $\uparrow$
}

\author{
Quan Xu, (D) *a Wei Cai, ${ }^{\text {a }}$ Miaoran Zhang, ${ }^{a}$ Rigu Su, ${ }^{a}$ Yingchun Ye, ${ }^{a}$ Yeqing $\mathrm{Li}{ }^{a}$ \\ Lipeng Zhang, ${ }^{* b}$ Yongjian Guo, ${ }^{b}$ Zhiqiang Yu, DD ${ }^{c}$ Siyu Li, ${ }^{c}$ Xun Lin, ${ }^{c}$ Yusheng Chen, ${ }^{d}$ \\ Yan Luo, ${ }^{e}$ Jason Street $^{f}$ and Meng $\mathrm{Xu}^{* \mathrm{~g}}$
}

Heteroatom-doped carbon dots (CDs) with excellent optical characteristics and negligible toxicity have emerged in many applications including bioimaging, biosensing, photocatalysis, and photothermal therapy. The metal-doping of CDs using various heteroatoms results in an enhancement of the photophysics but also imparts them with multifunctionality. However, unlike nonmetal doping, typical metal doping results in low fluorescence quantum yields (QYs), and an unclear photoluminescence mechanism. In this contribution, we detail results concerning zinc doped CDs (Zn-CDs) with QYs of up to $35 \%$. The zinc ion charges serve as a surface passivating agent and prevent the aggregation of graphene $\pi-\pi$ stacking, leading to an increase in the QY of the $\mathrm{Zn}-\mathrm{CDs}$. Structural and chemical investigations using spectroscopic and first principle simulations further revealed the effects of zinc doping on the CDs. The robust $\mathrm{Zn}$-CDs were used for the ultra-trace detection of $\mathrm{Hg}^{2+}$ with a detection limit of $0.1 \mu \mathrm{M}$, and a quench mechanism was proposed. The unique optical properties of the $\mathrm{Zn}-\mathrm{CDs}$ have promise for use in applications such as in vivo sensing and future phototherapy applications.

Received 30th March 2018
Accepted 26th April 2018

DOI: $10.1039 / \mathrm{c} 8 \mathrm{ra0} 2756 \mathrm{k}$

rsc.li/rsc-advances semi-crystalline CDs (S, N-CDs) with a QY of up to $55 \%$ have also been successfully produced. ${ }^{20}$ When compared to non-metal doped CDs, metal-ion doped CDs have been poorly explored. Metallic elements can serve as electron donors in CDs, boost electron transfer and introduce novel functionalities into the CDs. For example, Wu et al. reported that $\mathrm{Cu}$ could serve as an electron transfer booster in CDs and be used in photocatalysis. ${ }^{21}$ Bourlinos et al. created Gd(III)-doped CDs that could be used as dual fluorescence-MRI probes for biomedical applications. ${ }^{22}$ Thus far, most metal-doped CDs have shown a low PLQY. An indepth understanding of the PL mechanism of CDs is beneficial for tuning their fluorescence properties, which in turn can maximize their application.

Factors including the CD size effect, the surface passivation functionalization and the conjugate effect are considered to be important factors in tuning the PLQY of CDs. However, the explanations behind the mechanism responsible for PLQY remain contradictory. ${ }^{23-27}$ The photoluminescence (PL) properties of CDs vary with size, with small, medium, and large CDs emitting UV, visible light and near-infrared wavelengths, respectively. ${ }^{28}$ Guo et al. reported that the tuneable PL mechanism of CDs was due to the variations in the surface state affected by the $\mathrm{N}$ content. Yang et al. summarized PL mechanisms in a review by comparing graphene quantum dots, carbon nanodots, and polymer dots. This comparison included a description of the quantum confinement effect or conjugated d-domains, the surface state, the molecule state and the crosslink enhanced emission (CEE) effect. ${ }^{29}$ Thus far, the mechanism $\dagger$ Electronic supplementary information (ESI) available. See DOI: $10.1039 / \mathrm{c} 8 \mathrm{ra02756k}$ 
of metal-ion doped CDs has remained unclear. Metal doping may enhance both the electron donating/accepting abilities and can change CD surface charges, so further analysis of the QY of metal-doped CDs is crucial.

In this contribution, we analysed zinc-doped CDs to determine how the CD size and doping amount affected the PLQY of the CDs. The zinc ion charges serve as an important surface passivate agent and prevent the aggregation of the graphene $\pi-$ $\pi$ stacking. This led to the formation of sp3/sp2 hybrid hybridization, which is a key step in increasing the QY of ZnCDs. The prepared Zn-CDs had a QY of up to $35 \%$, and the first principles calculations further proved this hypothesis. These findings can effectively guide scientists in the preparation of high QY metal-doped CDs for applications in the biomedical field.

\section{Experimental section}

\section{Materials}

Sodium citrate, citric acid, mercuric nitrate, hydrogen peroxide and metal salts used in the detecting application section were purchased from Aladdin Industrial Inc. Zinc chloride used for doping was obtained from Tianjin Guangfu Technology Development Co., Ltd. All solutions were prepared using deionized water obtained from equipment bought from Dongguanshi Nabaichuan Water-Treatment Equipment Co., Ltd.

\section{Synthesis of the Zn-CDs}

Zinc-doped carbon dots (Zn-CDs) were prepared using a facile one-step hydrothermal method, which was optimized in our previous work. ${ }^{30}$ First, $25 \mathrm{~mL}$ of sodium citrate $(0.1 \mathrm{M})$ was mixed with zinc chloride $(0.05 \mathrm{M})$ and the mixture was poured into a $50 \mathrm{~mL}$ Teflon-lined stainless-steel autoclave. Then, samples of the product were placed in a drying cabinet at $185^{\circ} \mathrm{C}$ for a reaction time varying from $1 \mathrm{~h}$ to $10 \mathrm{~h}(1 \mathrm{~h}, 2 \mathrm{~h}, 4 \mathrm{~h}, 6 \mathrm{~h}, 8 \mathrm{~h}$, $10 \mathrm{~h}$ ). The products were first filtered to remove carbon impurities, salts or other impurities. Then, the product solutions were further purified over a number of days using a dialysis membrane filter $(0.22 \mathrm{~mm})$ against ultrapure water, which was renewed every 10-12 h, until no $\mathrm{Zn}$ was detected in the ultrapure water.

\section{Characterization}

A FLS980 Fluorescence Spectrometer from Techcomp (China) Ltd was used to measure the photoluminescence quantum yield. The morphology of the prepared CDs was investigated using transmission electron microscopy (TEM) (Model JEM2100). The atomic force microscopy (AFM) images were measured using a Bruker Scan-Dimension-Icon System. The ultraviolet-visible (UV-Vis) absorption spectra of the samples were measured using a UV-Vis spectrometer (Jasco V-570). A Bruker Vertex 70v was employed for the measurement of the Fourier transform infrared (FTIR) spectra. The fluorescence measurements were carried out using a Cary Eclipse fluorescence spectrophotometer (Varian, Inc.). The solutions of the prepared CDs were placed in a quartz fluorescence cuvette. The emission slits and excitation slits were set at $2.5 \mathrm{~nm}$ and $5 \mathrm{~nm}$, respectively. Elemental analysis was performed using X-ray photoelectron spectroscopy (XPS) (ESCALAB 250 spectrometer). Raman spectroscopy was performed by employing a Horiba Jobin Yvon Xplora confocal Raman microscope at room temperature.

\section{Simulation method}

Density functional theory (DFT) was employed to study the electronic properties of the zinc-doped carbon dots (Zinc-CD). We chose the B3LYP hybrid method with the 6-311G basis set within the Gaussian 09 package to calculate the optimized structure of the $\mathrm{Zn}-\mathrm{CDs}$ and their electronic properties. Three different sizes of $\mathrm{Zn}-\mathrm{CDs}\left(\mathrm{C}_{18} \mathrm{H}_{11} \mathrm{Zn}, \mathrm{C}_{24} \mathrm{H}_{13} \mathrm{Zn}\right.$ and $\left.\mathrm{C}_{30} \mathrm{H}_{15} \mathrm{Zn}\right)$ were built representing the growing of the $\mathrm{Zn}$-CDs at different reaction times ranging from a small size to a large size and the HOMO-LUMO of each model was calculated and discussed.

\section{Detection of $\mathbf{H g}^{2+}$}

The typical steps of the detecting experiment were as follows: 20 $\mu \mathrm{L}$ of the prepared $\mathrm{Zn}$-CDs were placed into $2 \mathrm{~mL}$ of deionized water, and then the fluorescence intensity at $440 \mathrm{~nm}$ of the $\mathrm{Zn}$ CDs (excited by light at $340 \mathrm{~nm}$ ) was measured and recorded as the initial fluorescence intensity $\left(F_{0}\right)$. Then, different concentrations of $\mathrm{Hg}^{2+}$ were introduced into the above sample solution. The fluorescence intensity was measured after $5 \mathrm{~min}$ and recorded as the fluorescence intensity of the end point $\left(F_{t}\right)$. The change in the fluorescence intensity $(\Delta F)$ was defined as the difference between $F_{0}$ and $F_{t}\left(\Delta F=F_{0}-F_{t}\right)$. The linear relationship between the change in the fluorescence intensity $\Delta F$ $\left(\Delta F=F_{0}-F_{1}\right)$ and the concentration of $\mathrm{Hg}^{2+}$ was determined.

To explore the selectivity of the $\mathrm{Hg}^{2+}$ detection, $50 \mu \mathrm{M}$ solutions of various metal ions (including $\mathrm{Hg}^{2+}, \mathrm{K}^{+}, \mathrm{Mg}^{2+}, \mathrm{Na}^{+}, \mathrm{Mn}^{2+}$, $\mathrm{Cu}^{+}, \mathrm{Cu}^{2+}, \mathrm{Al}^{3+}, \mathrm{Fe}^{2+}, \mathrm{Fe}^{3+}, \mathrm{Cl}^{-}, \mathrm{SO}_{4}{ }^{2-}$ and $\mathrm{NO}_{3}{ }^{-}$) were prepared using deionized water. Then $20 \mu \mathrm{L}$ of each of the sample solutions were added to the aforementioned solution $(2 \mathrm{~mL})$, respectively, and the fluorescence intensity of the mixtures was measured and defined as $F_{t}$. The change in the fluorescence intensity, $\Delta F$, was defined as: $\Delta F=F_{0}-F_{t}$.

\section{Results and discussion}

\section{Optimization of the synthesis conditions}

The prepared Zn-CDs solution was observed to be a lavender colour under visible light and exhibited a bright blue luminescence when excited by light at $365 \mathrm{~nm}$, as illustrated in Fig. 1a. The structure of the Zn-CDs was explored by transmission electron microscopy (TEM). Fig. 1a shows that the Zn-CDs were successfully synthesized by the one-step hydrothermal method, and the TEM showed the Zn-CDs to take the form of nanoparticles. The TEM images exhibited that there was an excellent separation of the particles, with a diameter range from 3-10 nm. The AFM images further demonstrated the formation of mostly monodispersed, quasi-spherical Zn-CDs with an average diameter of smaller than $3 \mathrm{~nm}$, devoid of any aggregation (Fig. $\mathrm{S} 2 \dagger$ ). 
a)

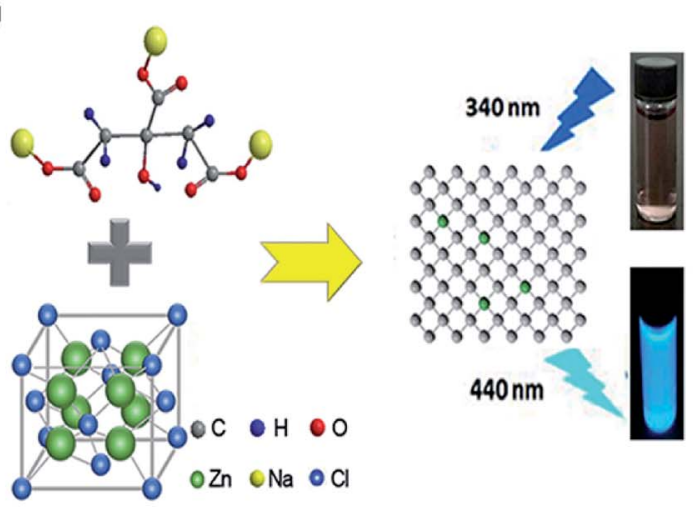

c)

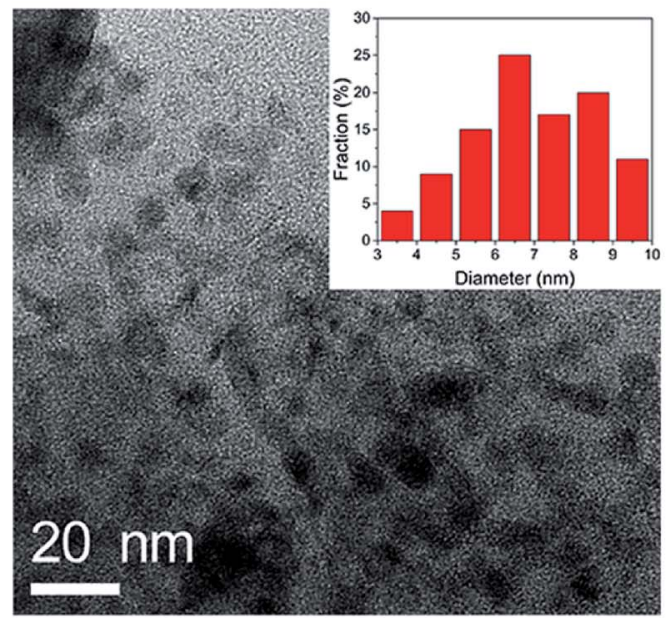

b)

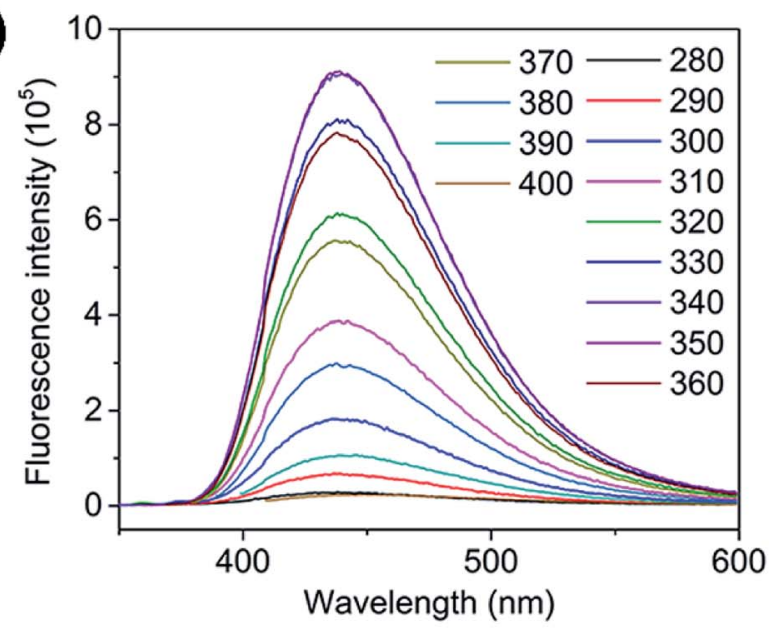

d)

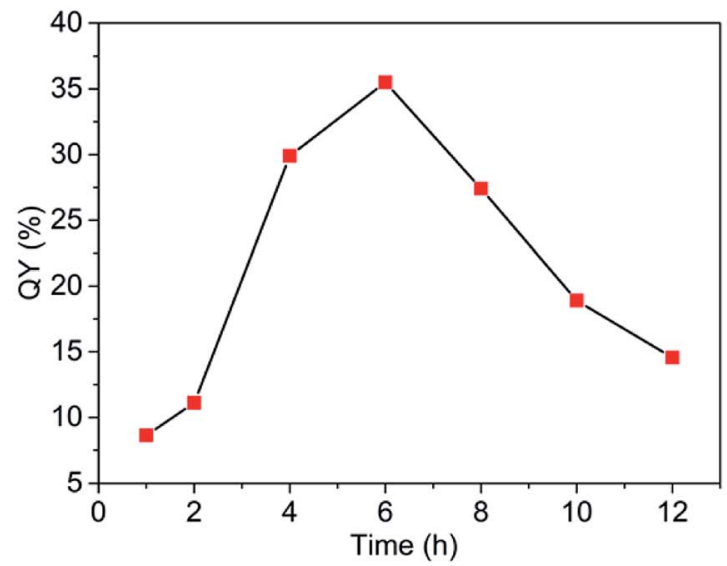

Fig. 1 (a) Schematic diagram of the synthesis of the $Z n-C D s$ with blue luminescence (excited by light at 340 nm); (b) photoluminescence spectra of the $\mathrm{Zn}-\mathrm{CDs}$; (c) TEM images of the $\mathrm{Zn}-\mathrm{CDs}$ and the diameter distribution of the $\mathrm{Zn}-\mathrm{CDs}$; (d) quantum yield of the $\mathrm{Zn}$ - $\mathrm{CDs}$ for reaction times varying from $1 \mathrm{~h}$ to $12 \mathrm{~h}(1 \mathrm{~h}, 2 \mathrm{~h}, 4 \mathrm{~h}, 6 \mathrm{~h}, 8 \mathrm{~h}, 10 \mathrm{~h}, 12 \mathrm{~h}$ ).

The photoluminescence characteristics of the synthesized $\mathrm{Zn}$-CDs were investigated in detail using a fluorescence spectrometer, which irradiated the samples at different wavelengths to analyze the emission properties. The prepared $\mathrm{Zn}$-CDs had the strongest emission, centred at $440 \mathrm{~nm}\left(\lambda_{\mathrm{em}}\right)$ when excited at a wavelength of $340 \mathrm{~nm}\left(\lambda_{\text {ex }}\right)$. This result is shown in Fig. $1 \mathrm{~b}$ and S1. $\dagger$ The variation of $\lambda_{\text {ex }}$ from 280 to $400 \mathrm{~nm}$ for the prepared samples only led to a rapid decrease in the intensity of $\lambda_{\mathrm{em}}$, without a shift in the peak position, which was shown in our previous studies..$^{30}$ Fig. $1 \mathrm{~d}$ shows that the quantum yield (QY) of the $\mathrm{Zn}$-CDs varied relative to the heating time. The prepared $\mathrm{Zn}$ CDs heated for $6 \mathrm{~h}$ had the highest quantum yield of $34.6 \%$. Fig. S3 $\uparrow$ shows that the fluorescence lifetime of the Zn-CDs was measured to be $6.32 \mathrm{~ns}$ using the time-correlated single photon counting (TCSPC) method. The results showed that the lifetime of the $\mathrm{Zn}$-CDs underwent almost no change as the reaction time increased, indicating little variety in the surface functional groups of the Zn-CDs.

In the C1s spectra (Fig. 2a), the two peaks at $288.5 \mathrm{eV}$ and $284.5 \mathrm{eV}$ are representative peaks of the $\mathrm{C}=\mathrm{O}$ and $\mathrm{C}-\mathrm{C}$ groups.
As the reaction time increased, the concentration of the $\mathrm{C}=\mathrm{O}$ groups first increased and then decreased, indicating that the carbon dots experienced maximum oxidation after approximately 4 hours. This phenomenon was also observed in the O1s spectra (Fig. 2b). The carbonyl peak also exhibited an initial increase and then a decrease as the reaction continued, which was consistent with what was shown in the C1s spectra. Both results show that after undergoing a reaction for $2-4 \mathrm{~h}$, the oxidation of the carbon dots reached its peak. This conclusion was further proved by the Raman spectra, shown in Fig. 2e. The $\mathrm{D}$ band is the indicator of the oxidation defect on the carbon dot surface and it exhibited a similar trend as seen in the C1s and O1s spectra. When normalized in the G band $\left(1600 \mathrm{~cm}^{-1}\right)$, the D band increased at the beginning of the reaction $(1,2,4 \mathrm{~h})$, and decreased as the reaction continued $(8,10 \mathrm{~h})$. According to the theory of passivation, a high $\mathrm{D} / \mathrm{G}$ ratio implies that there is a high surface defect/passivation ratio, which can serve as an excitation energy trap and increase the photoluminescence. This provides a good explanation for the QY change seen in Fig. 1d. The effect of an extreme environment, such as highly 
a)

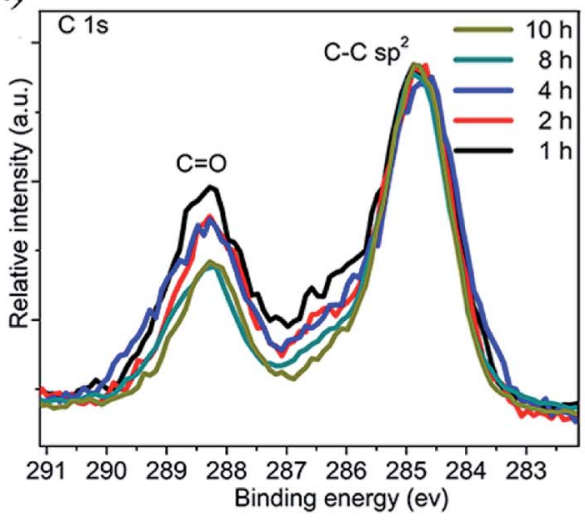

c)

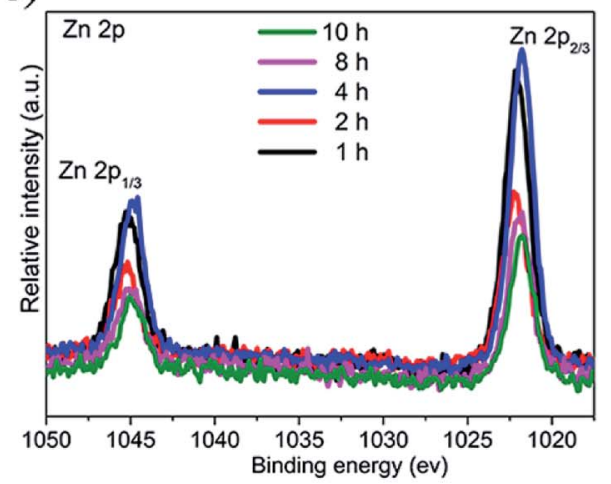

e)

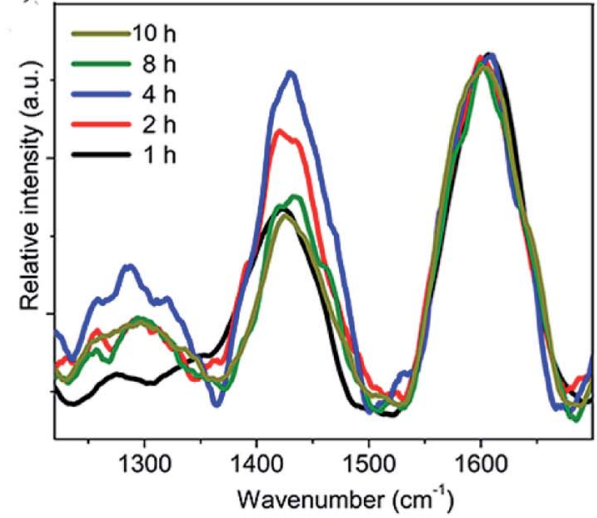

b)

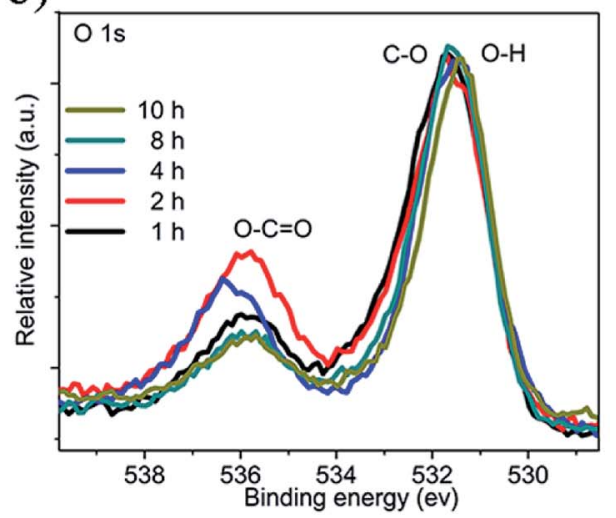

d)

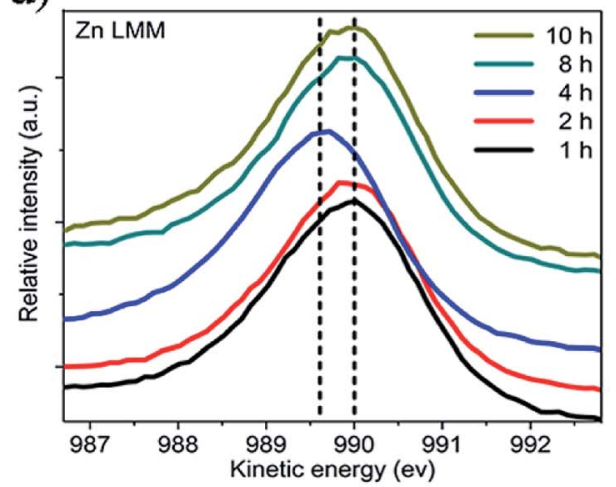

f)

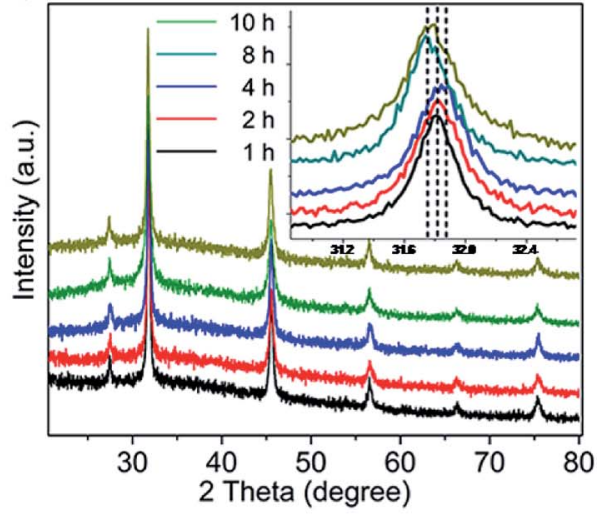

Fig. 2 (a) C1s, (b) O1s, (c) Zn2p, and (d) Zn LMM XPS spectra of the Zn-CDs; (e) Raman spectra and (f) PXRD patterns of the Zn-CDs.

oxidizing solutions (hydrogen peroxide), was also tested and the Zn-CDs manifested high stability and steady fluorescence against highly oxidizing $\mathrm{H}_{2} \mathrm{O}_{2}$ (Fig. $\mathrm{S} 4 \dagger$ ).

The $\mathrm{Zn}$ peak in the $\mathrm{Zn}$ LMM spectra shifted to a higher binding energy in the first part of the reaction and then shifted back to a lower binding energy (Fig. 2d). This indicated that during the growth phase of the carbon dots, that $\mathrm{Zn}$ was firstly oxidized and then reduced. These results combined with the 01s and C1s spectra showed a high possibility that the high oxidation state of the $\mathrm{Zn}$ was connected to the carbonyl group. It was observed that 2 theta in the Zn PXRD patterns exhibited a slight shift during the reaction time of 2-4 hours, indicating the change in the lattice distance, which may have resulted from the high oxidation state of the Zn (Fig. 2f). The crystal structure of $\mathrm{Zn}$-CDs was a lattice structure, which indicated an existence of crystallization characteristics of graphitic carbon due to a sp2 hybridization (Fig. 3a-c). The lattice fringe spacing is shown in Fig. 3c, of which the interplanar distance of $0.22 \mathrm{~nm}$ corresponded to the (002) plane of Zn-CDs. It was observed that the images of the particles were darker, which indicated the growth of the crystalline Zn-CDs (Fig. 3d). Fig. 3b shows the areas partitioned by dotted lines had the existence of both the lattice structure and absolute amorphous structure. The existence of the amorphous structure indicated a sp3/sp2 hybrid 
hybridization, indicating the formation of a 3D structure. Considering that the high oxidation state of the $\mathrm{Zn}$ concurred with the high oxidation of the carbon dots, it is reasonable to assume that $\mathrm{Zn}$ may play an important role as the active binding site to connect neighbouring small carbon dots through chelation between $\mathrm{Zn}$ and the carbonyl group. This process is believed to indirectly promote the growth of large carbon dots (Fig. 3d). The change in the peaks in the UV-Vis absorption spectra (Fig. S5 $\dagger$ ) provided strong evidence that the cyclization degree of the $\mathrm{Zn}$-CDs rose as the reaction progressed. The IR spectra in Fig. S6† show the existence of $\mathrm{OH}$ groups inside the
Zn-CDs, which corresponds to what is shown in the XPS results of O1s. It is assumed that the better solubility of the Zn-CDs in water was due to the polar $\mathrm{OH}$ groups on the surface of the CDs, which could form intermolecular hydrogen bonds in the solution.

\section{Simulations}

To further understand the Zn doping process and its effect on the CDs, we proposed a theoretical lattice model of the Zn-CDs. Both the structural and electronic properties were investigated as a)

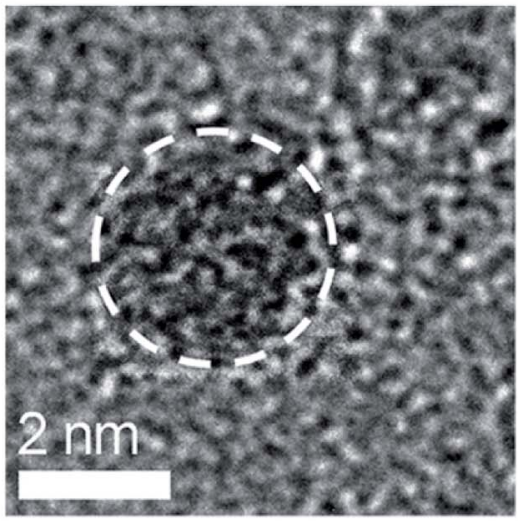

c)

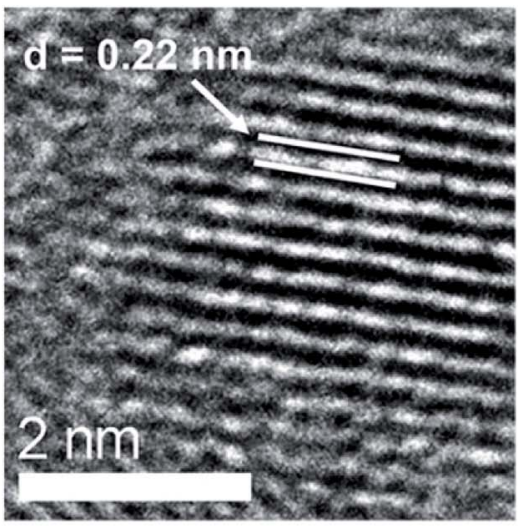

b)

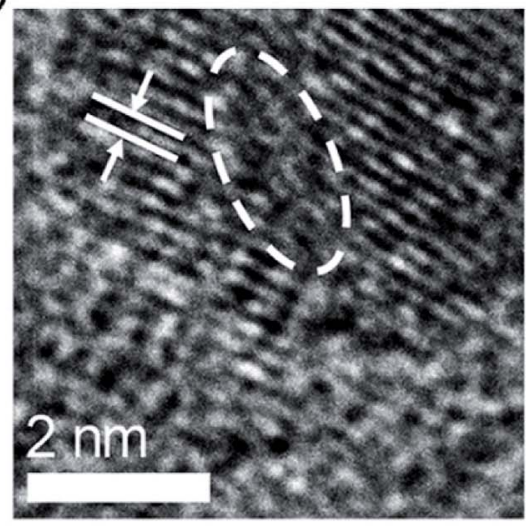

d)

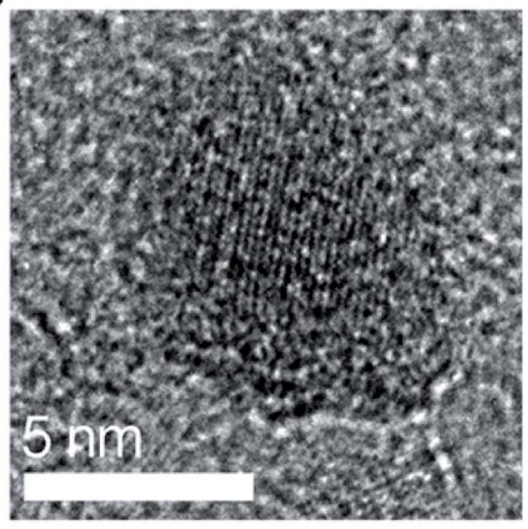

e)

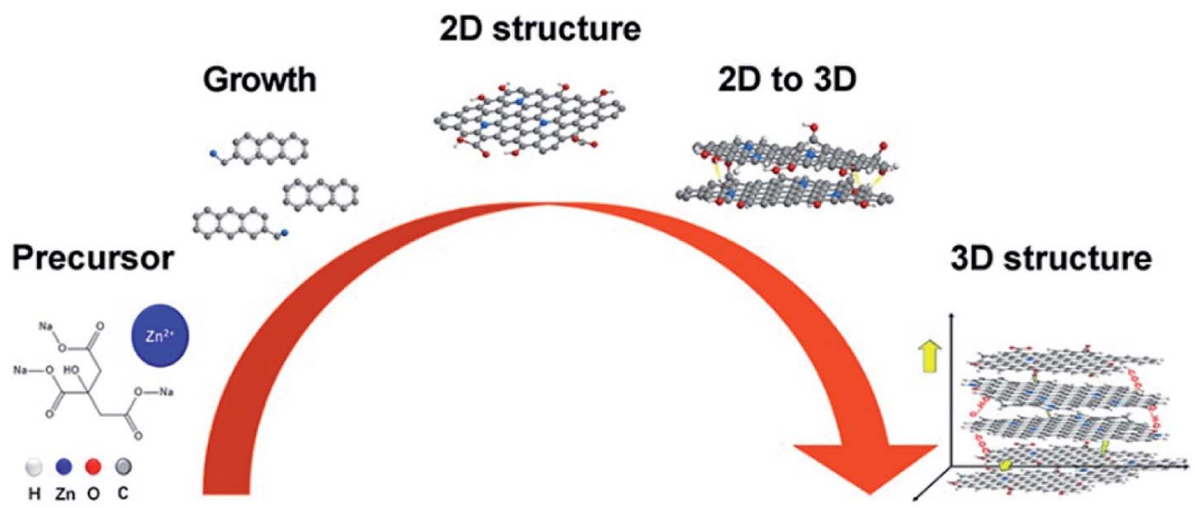

Fig. 3 (a-d) TEM images of the $\mathrm{Zn}-\mathrm{CD}$ s with reaction times of $1 \mathrm{~h}, 2 \mathrm{~h}, 4 \mathrm{~h}$ and $10 \mathrm{~h}$, respectively. The cross lattices show the 3D structure of the $\mathrm{Zn}$-CDs. (e) A schematic diagram showing the growth of the $\mathrm{Zn}-\mathrm{CD}$ s from a sp2 to a sp3 structure. 

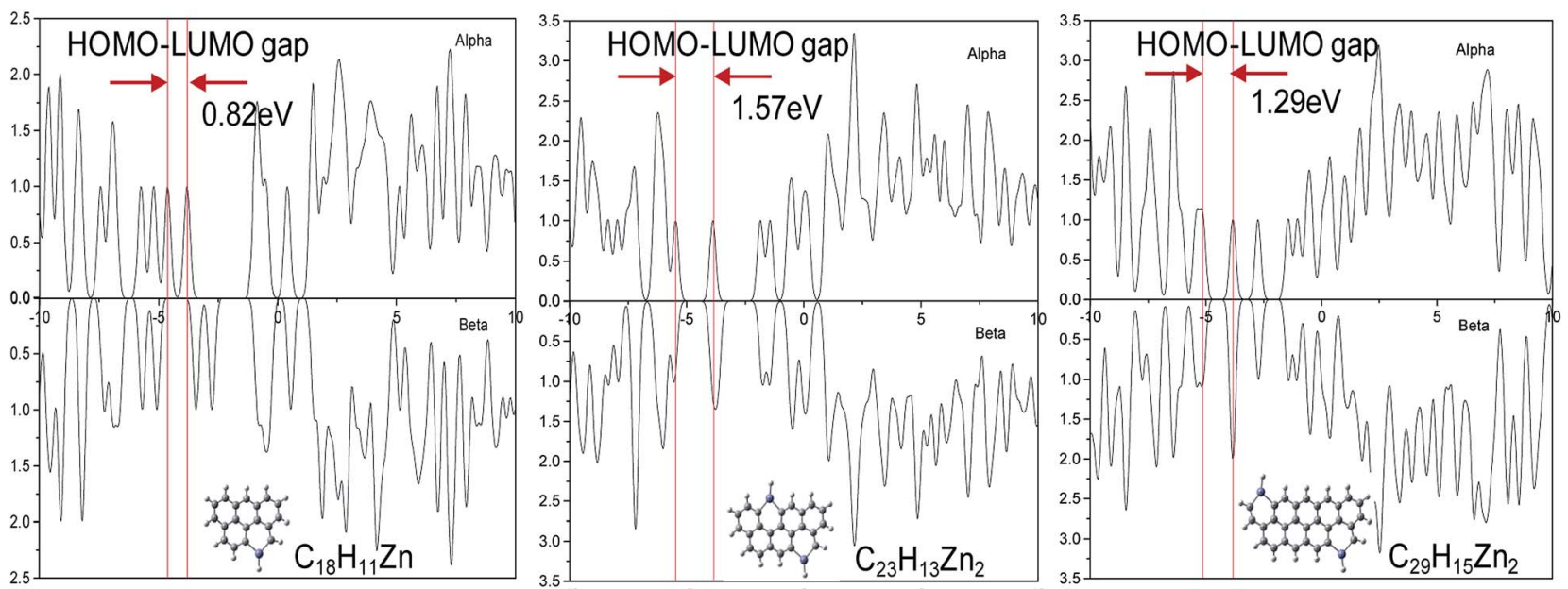

Fig. 4 Density of states for the different sizes of $Z n-C D s$.

functions of the location of impurity/dopant atoms incorporated into the CDs. Our results clearly showed that the location of the $\mathrm{Zn}$ atoms incorporated into the $\mathrm{CD}$ lattice plays an important role in regulating the properties of the doped CDs. The HOMOLUMO energy gap of the three Zn-CD materials were calculated and were found to have values of $0.82 \mathrm{eV}, 1.57 \mathrm{eV}$ and $1.29 \mathrm{eV}$ as the size of the Zn-CDs increased, as shown in Fig. 4. Among these three Zn-CDs, the concentration of oxidized Zn first increased and then decreased. The results clearly revealed that when comparing pristine-CDs with the Zn-CDs, that the doped $\mathrm{Zn}$ ions tune the HOMO-LUMO values and the energy gap. In three different sizes of pristine-CDs, the HOMO-LUMO energy gaps were found to be $\sim 1.75 \mathrm{eV}$, as listed in Table 1 . The doped $\mathrm{Zn}$ adjusts the HOMO-LUMO gap of the $\mathrm{C}_{23} \mathrm{H}_{13} \mathrm{Zn}_{2}$ to a value of $1.57 \mathrm{eV}$, in which the concentration of the oxidized $\mathrm{Zn}$ group is neither lower nor higher than $1.57 \mathrm{eV}$. This value may correspond to the preferred radiation from the singlet excitation state $S_{1}$ to the ground state $S_{0}$, therefore generating the high fluorescence QY. The simulation results revealed that doped Zn ions play an important role as the CDs grow from a small size to a medium size. They may increase charge transfer/charge recombination and thus increase the QY of the CDs with a certain concentration. However, the doping would cause a loss in function as the CDs kept growing as more graphene would $\pi-\pi$ stack, and thus cause the QY of the CDs to decrease.

\section{Detection of $\mathbf{H g}^{2+}$}

To explore the potential application of Zn-CDs, fluorescence probes adopting Zn-CDs were studied for their detection of $\mathrm{Hg}^{2+}$. The environmental hazards of mercury can eventually impact upon plants, animals, and human health due to its bioaccumulation. Mercury in the environment can be transferred through the food chain and greatly threaten human health. Even very low concentrations can cause diseases including kidney failure, permanent brain damage, and various motion and cognitive disorders. ${ }^{31,32}$ The most frequently used technologies for $\mathrm{Hg}^{2+}$ detection, ultraviolet-visible spectroscopy (UV-Vis) and inductively coupled plasma mass spectrometry
(ICP-MS), usually demands expensive equipment and complicated procedures. Here, we report a fluorescence-based detection method, which could be employed for $\mathrm{Hg}^{2+}$ detection with good sensitivity, instant response, high selectivity, and simple operation.

In this report, synthesized Zn-CDs were found to be efficient for detecting $\mathrm{Hg}^{2+}$. Once $10 \mu \mathrm{M}$ of $\mathrm{Hg}^{2+}(20 \mu \mathrm{L})$ was introduced to the $\mathrm{Zn}$-CDs, an absolute drop in the fluorescence intensity was observed (Fig. 5a). Through an alternate and efficient electron transfer process, $\mathrm{Hg}^{2+}$ caused the efficient quenching of $\mathrm{Zn}$-CDs due to its ability to facilitate electron/hole recombination annihilation. When the $\mathrm{Hg}^{2+}$ solution was introduced, a substantial decay was observed. Fig. $5 \mathrm{~b}$ and $\mathrm{c}$ show the trend in the decay of the fluorescence intensity of the Zn-CDs as the concentrations of $\mathrm{Hg}^{2+}$ increased. Each spectrum was recorded after $5 \mathrm{~min}$ to keep an equilibration reaction time. A linear relationship was found between the $\mathrm{Hg}^{2+}$ concentration and the fluorescence intensity of the Zn-CDs. The resultant calibration curve for the $\mathrm{Hg}^{2+}$ had excellent linearity in a concentration range of 1 to $60 \mu \mathrm{M}$, with a coefficient of determination of 0.9894 (Fig. 5c). The limit of detection was $100 \mathrm{nM}$. To explore the quenching selectivity of the $\mathrm{Zn}-\mathrm{CDs}$ for $\mathrm{Hg}^{2+}$ as a sensing probe, the detection was carried out after introducing various metal ions. In Fig. 5 d, it can be seen that $\mathrm{Hg}^{2+}$ had the highest influence on the fluorescence intensity of the Zn-CDs among all of the metal ions. Moreover, the changes in the lifetime (Fig. 5e) and absorption (Fig. 5f) provided evidence that the change in the surface state of the $\mathrm{Zn}$-CDs was brought about by $\mathrm{Hg}^{2+}$.

Table 1 The HOMO-LUMO energy gaps of the $\mathrm{Zn}-\mathrm{CDs}$

\begin{tabular}{llll}
\hline $\mathrm{Zn}-\mathrm{CDs} / \mathrm{CDs}$ & $E_{\text {номо }}(\mathrm{eV})$ & $E_{\text {LUмо }}(\mathrm{eV})$ & $\Delta E_{\text {gap }}(\mathrm{eV})$ \\
\hline $\mathrm{C}_{18} \mathrm{H}_{11} \mathrm{Zn}$ & -4.61 & -3.79 & 0.82 \\
$\mathrm{C}_{19} \mathrm{H}_{11}$ & -4.34 & -2.59 & 1.75 \\
$\mathrm{C}_{23} \mathrm{H}_{13} \mathrm{Zn}_{2}$ & -3.89 & -5.46 & 1.57 \\
$\mathrm{C}_{25} \mathrm{H}_{13}$ & -4.37 & -2.64 & 1.73 \\
$\mathrm{C}_{29} \mathrm{H}_{15} \mathrm{Zn}_{2}$ & -5.12 & -3.83 & 1.29 \\
$\mathrm{C}_{31} \mathrm{H}_{15}$ & -4.41 & -2.66 & 1.75
\end{tabular}


a)

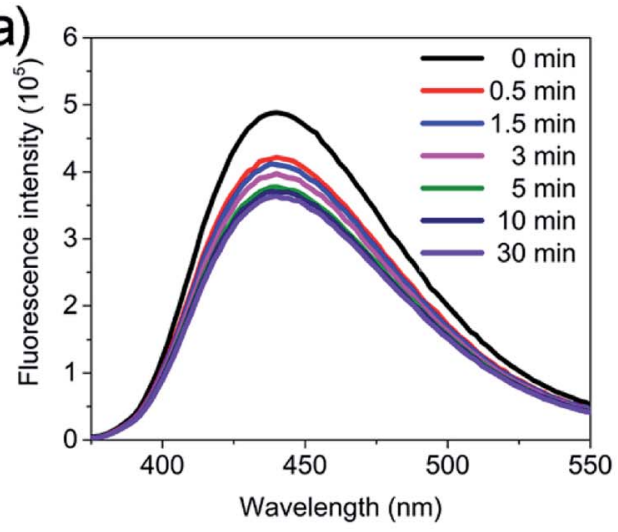

c)

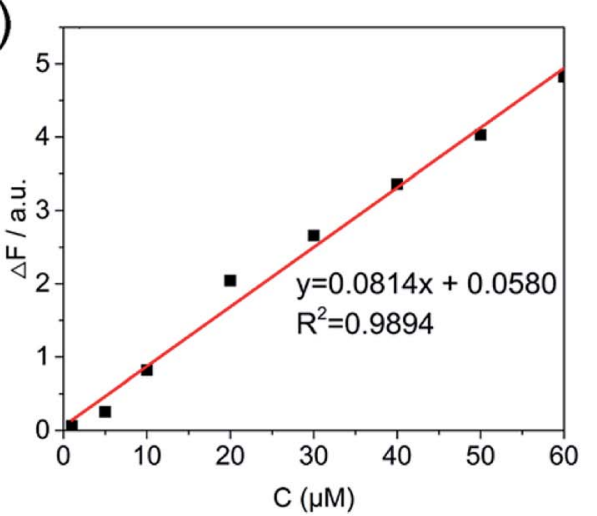

e)

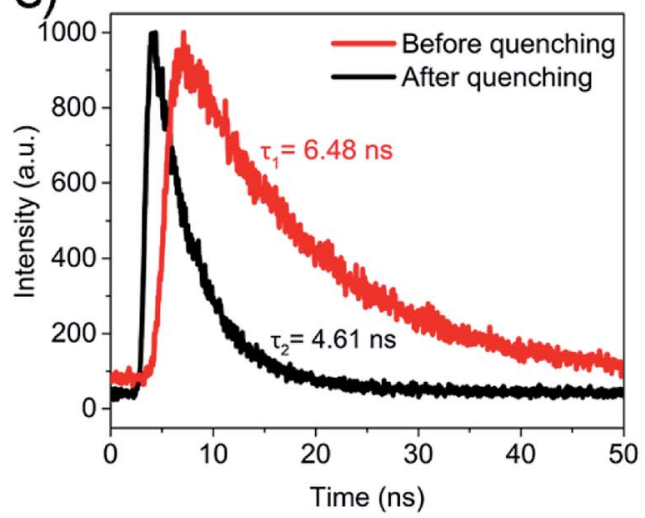

b)

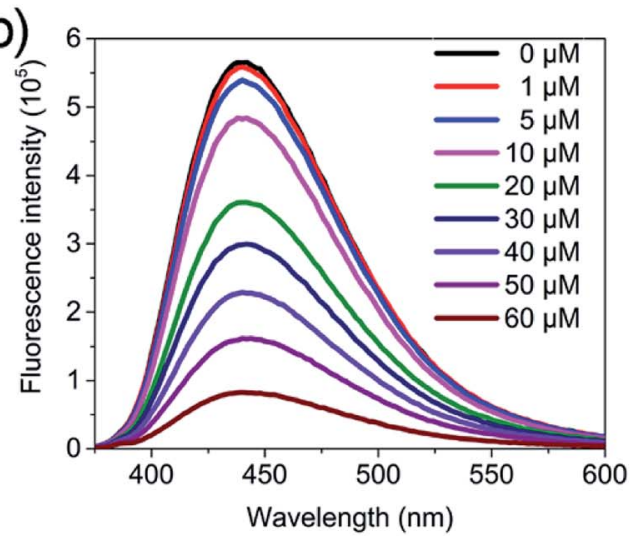

d)

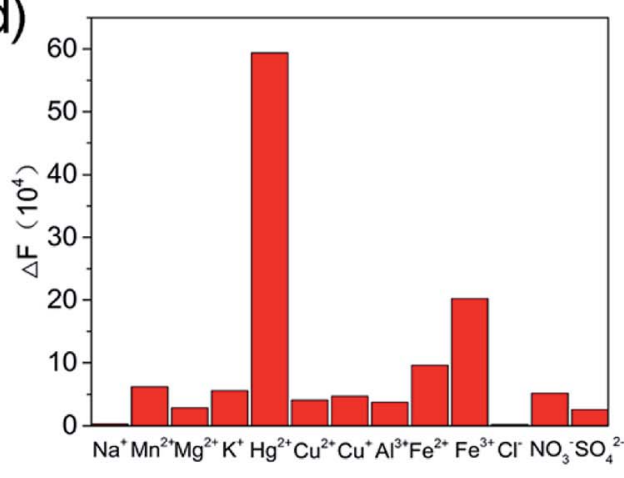

f)

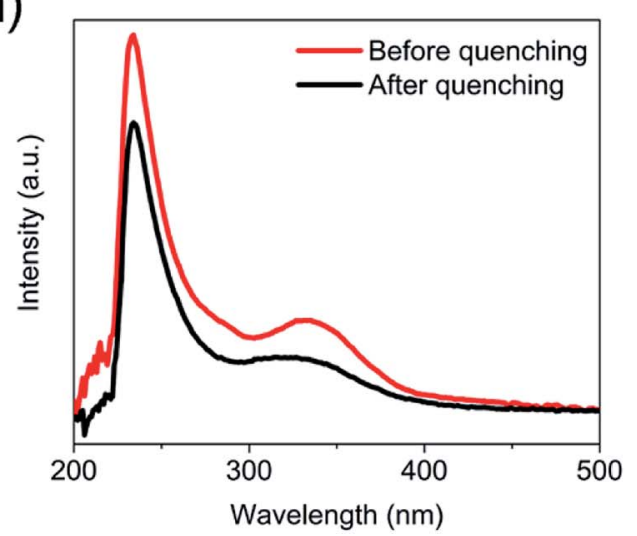

Fig. 5 (a) Time-dependent fluorescence changes of the $\mathrm{Zn}$-CDs in the presence of $\mathrm{Hg}^{2+}(10 \mu \mathrm{M})$; (b) PL emission spectra of the Zn-CDs solution with different concentrations of $\mathrm{Hg}^{2+}(0,1,5,10,20,30,40,50,60 \mu \mathrm{M})$; (c) $\Delta F$ calibration curve of the $\mathrm{Zn}$-CDs solution versus the concentration of $\mathrm{Hg}^{2+}$; (d) the $\Delta F$ at $440 \mathrm{~nm}$ for the $\mathrm{Zn}-\mathrm{CDs}$ in the presence of various metal ions and anions (50 $\mu \mathrm{M}$ ); (e) lifetimes and (f) absorption spectra before and after the quenching of $\mathrm{Zn}-\mathrm{CDs}$ by $\mathrm{Hg}^{2+}$.

There was a complexation as $\mathrm{Hg}^{2+}$ was introduced, which caused electron or energy transfer. ${ }^{33,34}$ Thus, the Zn-CDs were found to have a good specificity for the detection of $\mathrm{Hg}^{2+}$. Based on the quenching effect of mercury ions on the fluorescence of CQDs, our group previously used S, N-doped CDs for the selective and sensitive sensing of $\mathrm{Hg}^{2+}$ in the aqueous phase with a limit of detection (LOD) of $100 \mathrm{pM} .{ }^{20}$ As for metal-doped $\mathrm{CDs}$, Liu et al. reported $\mathrm{Mg}, \mathrm{N}$-doped CDs for the sensing of $\mathrm{Hg}^{2+}$ with a LOD of $0.2 \mu \mathrm{M},{ }^{35}$ and Yuan et al. reported Ge-doped CDs with a LOD of $0.075 \mu \mathrm{M} .^{36}$ However, the $\mathrm{Zn}$-CDs we have reported showed a LOD of $0.1 \mu \mathrm{M}$, which is well placed among the LOD values of the aforementioned materials. It shows the great potential of the $\mathrm{Zn}$-CDs for application in sensing and detecting. Moreover, the PL properties of the $\mathrm{Zn}-\mathrm{CDs}$ in $0.1 \mathrm{M} \mathrm{H}_{2} \mathrm{O}_{2}$ and water (Fig. S6 $\dagger$ ) were investigated and found to be quite stable. The results show that the Zn-CDs have excellent potential for use in the protection and monitoring of the environment.

\section{Conclusions}

In this report, we first determined how the heating time affects the growth of Zn-CDs. The degree of graphitization had a non-negligible effect on the fluorescence mechanism 
and PLQY of the Zn-CDs. The XPS results showed the formation of $\mathrm{C}-\mathrm{Zn}$ bonds in the $\mathrm{Zn}$-CDs, strongly proving that the successful doping of $\mathrm{Zn}$ contributed to the emission of the bright blue fluorescence. Moreover, the $\mathrm{Zn}$-CDs showed a good selectivity for $\mathrm{Hg}^{2+}$ with a detection limit of $0.1 \mu \mathrm{M}$. This shows that Zn-CDs could be employed in environmental monitoring. This work concerning the growth mechanism of Zn-CDs provides guidance for the controllable preparation and functional design of CDs. This study assists in determining the factors responsible for the advantageous synthesis, heteroatom doping, and functionalization of carbon dots for use in the environmental and biomedical fields.

\section{Conflicts of interest}

There are no conflicts to declare.

\section{Acknowledgements}

We thank the National Key Research and Development Plan (No. 2016YFC0303701), the Beijing Nova Program Interdisciplinary Studies Cooperative project (No. Z181100006218138), the Beijing Science and Technology project (No. Z161100001316010), the State Key Laboratory of Petroleum \& Petrochemical Pollution Control and Treatment (No. PPC2017015), the Science Foundation of the China University of Petroleum, Beijing (2462018BJC004) for support. This study is supported by the National Institute of Food and Agriculture, the U.S. Department of Agriculture, and McIntire Stennis under accession number 1009735.

\section{References}

1 S. N. Baker and G. A. Baker, Angew. Chem., Int. Ed. Engl., 2010, 49, 6726-6744.

2 S. Zhu, Q. Meng, L. Wang, J. Zhang, Y. Song, H. Jin, K. Zhang, H. Sun, H. Wang and B. Yang, Angew. Chem., Int. Ed. Engl., 2013, 125, 4045-4049.

3 J. Ge, M. Lan, B. Zhou, W. Liu, L. Guo, H. Wang, Q. Jia, G. Niu, X. Huang, H. Zhou, X. Meng, P. Wang, C. S. Lee, W. Zhang and X. Han, Nat. Commun., 2014, 5, 4596-4603.

4 B. Zhang, J. Wang, J. Yu, X. Fang, X. Wang and D. Shi, Bioconjugate Chem., 2017, 28, 330-335.

5 J. Wang, Y. Zhong, X. Wang, W. Yang, F. Bai, B. Zhang, L. Alarid, K. Bian and H. Fan, Nano Lett., 2017, 17, 69166921.

6 W. Yang, W. Guo, W. Le, G. Lv, F. Zhang, L. Shi, X. Wang, J. Wang, S. Wang, J. Chang and B. Zhang, ACS Nano, 2016, 10, 10245-10257.

7 F. Yuan, Z. Wang, X. Li, Y. Li, Z. a. Tan, L. Fan and S. Yang, Adv. Mater., 2017, 29, 1604436-1604441.

8 D. H. Kim and T. W. Kim, Nano Energy, 2017, 32, 441-447.

9 L. Cui, X. Wang, N. Chen, B. Ji and L. Qu, Nanoscale, 2017, 9, 9089-9094.

10 L. F. Cui, X. P. Wang, N. Chen, G. F. Zhang and L. T. Qu, J. Mater. Chem. A, 2017, 5, 14508-14513.
11 S. Fang, Y. Xia, K. Lv, Q. Li, J. Sun and M. Li, Appl. Catal., B, 2016, 185, 225-232.

12 Q. Han, B. Wang, J. Gao and L. Qu, Angew. Chem., Int. Ed. Engl., 2016, 55, 10849-10853.

13 X. Y. Kong, W. L. Tan, B.-J. Ng, S.-P. Chai and A. R. Mohamed, Nano Res., 2017, 10, 1720-1731.

14 P. Wang, X. Zhang, J. Zhang, S. Wan, S. Guo, G. Lu, J. Yao and X. Huang, Nat. Commun., 2017, 8, 14580-14588.

15 R. Wang, K.-Q. Lu, Z.-R. Tang and Y.-J. Xu, J. Mater. Chem. A, 2017, 5, 3717-3734.

16 J. Chen, J. S. Wei, P. Zhang, X. Q. Niu, W. Zhao, Z. Y. Zhu, H. Ding and H. M. Xiong, ACS Appl. Mater. Interfaces, 2017, 9, 18429-18433.

17 H. Li, X. He, Z. Kang, H. Huang, Y. Liu, J. Liu, S. Lian, C. H. Tsang, X. Yang and S. T. Lee, Angew. Chem., Int. Ed. Engl., 2010, 49, 4430-4434.

18 C. J. Reckmeier, J. Schneider, A. S. Susha and A. L. Rogach, Opt. Express, 2016, 24, A312-A340.

19 Q. Xu, P. Pu, J. Zhao, C. Dong, C. Gao, Y. Chen, J. Chen, Y. Liu and H. Zhou, J. Mater. Chem. A, 2015, 3, 542-546.

20 Q. Xu, Y. Liu, C. Gao, J. Wei, H. Zhou, Y. Chen, C. Dong, T. S. Sreeprasad, N. Li and Z. Xia, J. Mater. Chem. C, 2015, 3, 9885-9893.

21 W. Wu, L. Zhan, W. Fan, J. Song, X. Li, Z. Li, R. Wang, J. Zhang, J. Zheng, M. Wu and H. Zeng, Angew. Chem., Int. Ed. Engl., 2015, 54, 6540-6544.

22 A. B. Bourlinos, A. Bakandritsos, A. Kouloumpis, D. Gournis, M. Krysmann, E. P. Giannelis, K. Polakova, K. Safarova, K. Hola and R. Zboril, J. Mater. Chem., 2012, 22, 2332723330.

23 Q. Xu, T. Kuang, Y. Liu, L. Cai, X. Peng, T. Sreenivasan Sreeprasad, P. Zhao, Z. Yu and N. Li, J. Mater. Chem. B, 2016, 4, 7204-7219.

24 F. Zhao, L. Wang, Y. Zhao, L. Qu and L. Dai, Adv. Mater., 2017, 29, 1604972-1604978.

25 Q. Yang, Z. Ma, H. Wang, B. Zhou, S. Zhu, Y. Zhong, J. Wang, H. Wan, A. Antaris, R. Ma, X. Zhang, J. Yang, X. Zhang, H. Sun, W. Liu, Y. Liang and H. Dai, Adv. Mater., 2017, 29, 1605497-1605505.

26 S. Sun, L. Zhang, K. Jiang, A. Wu and H. Lin, Chem. Mater., 2016, 28, 8659-8668.

27 X. Li, Y. Liu, X. Song, H. Wang, H. Gu and H. Zeng, Angew. Chem., Int. Ed. Engl., 2015, 54, 1759-1764.

28 L. Guo, J. Ge, W. Liu, G. Niu, Q. Jia, H. Wang and P. Wang, Nanoscale, 2016, 8, 729-734.

29 S. Zhu, Y. Song, X. Zhao, J. Shao, J. Zhang and B. Yang, Nano Res., 2015, 8, 355-381.

30 Q. Xu, Y. Liu, R. Su, L. Cai, B. Li, Y. Zhang, L. Zhang, Y. Wang, Y. Wang, N. Li, X. Gong, Z. Gu, Y. Chen, Y. Tan, C. Dong and T. S. Sreeprasad, Nanoscale, 2016, 8, 1791917927.

31 H. H. Harris, I. J. Pickering and G. N. George, Science, 2003, 301, 1203.

32 E. M. Nolan and S. J. Lippard, Chem. Rev., 2008, 108, 34433480 .

33 Y. Dong, R. Wang, H. Li, J. Shao, Y. Chi, X. Lin and G. Chen, Carbon, 2012, 50, 2810-2815. 
34 L. Wang, B. Li, F. Xu, X. Shi, D. Feng, D. Wei, Y. Li, Y. Feng, Y. Wang, D. Jia and Y. Zhou, Biosens. Bioelectron., 2016, 79, 1-8.
35 T. Liu, N. Li, J. X. Dong, H. Q. Luo and N. B. Li, Sens. Actuators, B, 2016, 231, 147-153.

36 Y. H. Yuan, R. S. Li, Q. Wang, Z. L. Wu, J. Wang, H. Liu and C. Z. Huang, Nanoscale, 2015, 7, 16841-16847. 